Research Article

\title{
Preparation and Gas Separation Performance of Polysulfone Mixed Matrix Membrane
}

\author{
Lili Jiang $\mathbb{D},{ }^{1,2}$ Yimin Meng, ${ }^{3}$ Su Xu, ${ }^{3}$ Haitao Yu, ${ }^{4}$ and Xingang Hou ${ }^{1}$ \\ ${ }^{1}$ State Key Laboratory of Advanced Processing and Recycling of Non-ferrous Metals, Lanzhou University of Technology, Lanzhou, \\ Gansu 730050, China \\ ${ }^{2}$ Wenzhou Engineering Institute of Pump \& Valve, Lanzhou University of Technology, Wenzhou, Zhejiang 325000, China \\ ${ }^{3}$ School of Material Science and Technology, Lanzhou University of Technology, Langongping Road, Lanzhou, \\ 730050 Gansu Province, China \\ ${ }^{4}$ Department of Medical Laboratory, The First Hospital of Lanzhou University, No. 1, Donggang Road, Chengguan District, \\ Lanzhou, 730000 Gansu Province, China
}

Correspondence should be addressed to Lili Jiang; jianglili2002@163.com

Received 15 March 2021; Revised 20 August 2021; Accepted 15 September 2021; Published 13 October 2021

Academic Editor: Hassan Karimi-Maleh

Copyright (c) 2021 Lili Jiang et al. This is an open access article distributed under the Creative Commons Attribution License, which permits unrestricted use, distribution, and reproduction in any medium, provided the original work is properly cited.

\begin{abstract}
As an economical, environmentally friendly, and highly efficient separation technology, membrane separation is a popular research topic in the field of separation. Organic polymer materials have attracted considerable attention in membrane separation because of their controllable preparation processes, simple modification method, and high toughness. Taking polysulfone (PSF) as the substrate of gas separation membrane, we prepared the mixed matrix membrane jointly by using the solution casting method and by adding graphene oxide (GO) and carbon nanotubes (CNTs). On this basis, the permeability of the membrane for $\mathrm{CO}_{2}$ and $\mathrm{N}_{2}$ and the permeability coefficient of the mixed gas were studied. With the addition of CNTs and GO, the permeability of gas was significantly improved. At $0.2 \mathrm{MPa}$, permeability of $\mathrm{CO}_{2}$ increased from 553 Barrer to 975 Barrer, and permeability of $\mathrm{N}_{2}$ increased from 536 Barrer to 745 Barrer. The max ideal separation coefficient of $\mathrm{CO}_{2}$ and $\mathrm{N}_{2}$ is 1.94 at $0.1 \mathrm{MPa}$. Increasing of the content of carbon nanotubes can significantly improve the permeability coefficient of $\mathrm{CO}_{2}$, while the change of inlet side pressure has a great impact on the permeability coefficient of $\mathrm{N}_{2}$. At $0.1 \mathrm{MPa}$, when the ratio of CNTs to GO was $5: 1$, the ideal permeability coefficient of $\mathrm{CO}_{2} / \mathrm{N}_{2}$ was 1.94, whereas the ideal permeability coefficient of PSF membrane was 1.46 . The above results of PSF/GO/CNT mixed matrix membrane lay a theoretical foundation for industrial application.
\end{abstract}

\section{Introduction}

With the continuous development of science and technology, products are also upgraded and improved continuously in the traditional manufacturing and metal smelting industries. As a result, the demand for energy in related industries is also increasing [1-3]. Traditional energy products are mainly nonrenewable fossil energy, such as oil and coal. However, due to its limited reserves, the demand is unable to adapt to the current growing energy demand, and with the current continuous exploitation, the sharp decline in reserves has led to widespread concern and worry. Therefore, researchers focus on the development of new energy and the recycling of energy byproducts in the production process to solve the energy problem.
As an energy by-product, if the blast furnace gas produced in metal smelting can be recycled, then it can reduce the production cost of enterprises and save some energy, thereby facilitating green production. However, the main compositions of blast furnace gas are incombustible $\mathrm{N}_{2}$ and $\mathrm{CO}_{2}$, and the content of combustible $\mathrm{CO}$ is low in blast furnace gas. As a result, its calorific value is low, and it is difficult to recycle. Searching a feasible and effective way to separate $\mathrm{N}_{2}$ and $\mathrm{CO}_{2}$ from blast furnace gas is a feasible way to improve the utilization rate of blast furnace gas $[4,5]$.

The common gas separation methods include adsorption method, absorption method, membrane separation, and condensation gasification. Among them, because different gas molecules have different diffusion rates passing through 


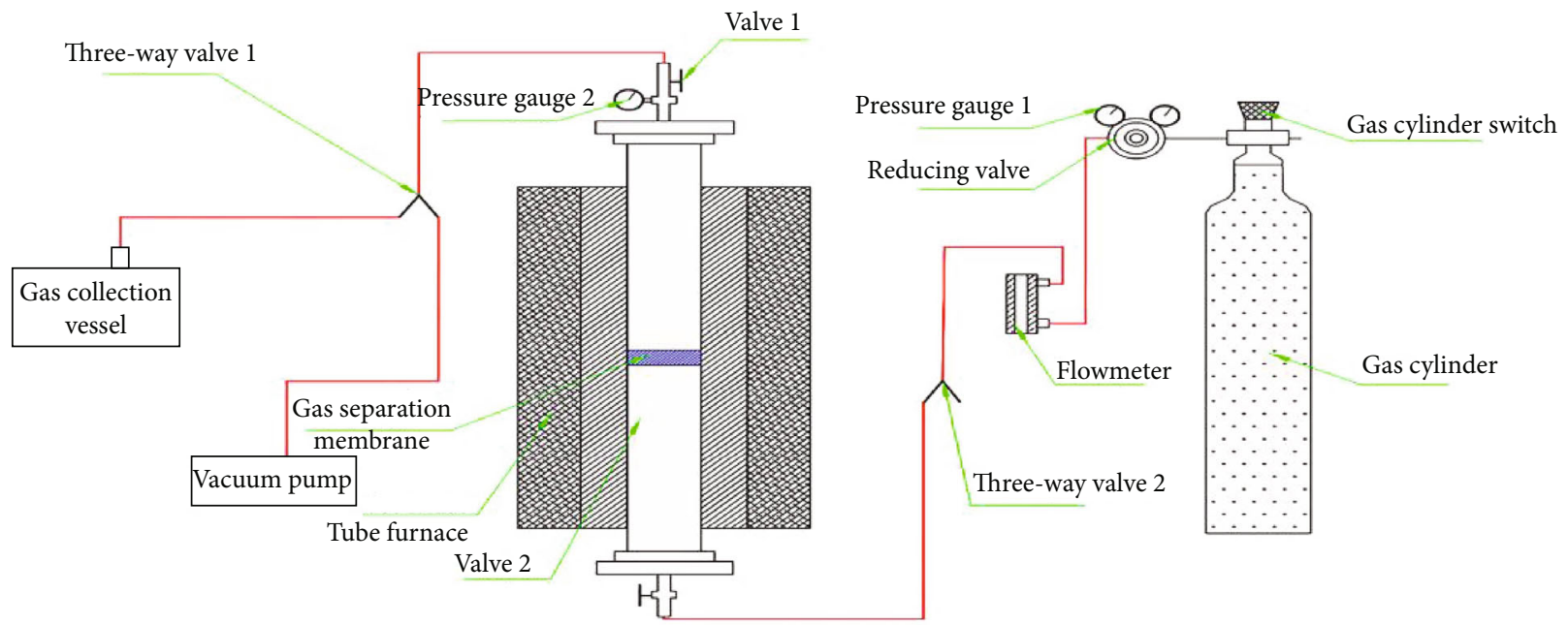

Figure 1: Experimental apparatus for gas separation experiment.

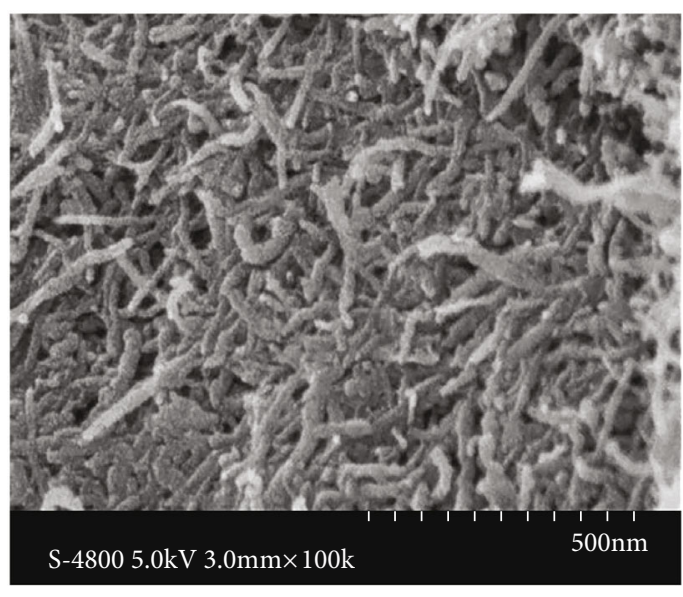

(a)

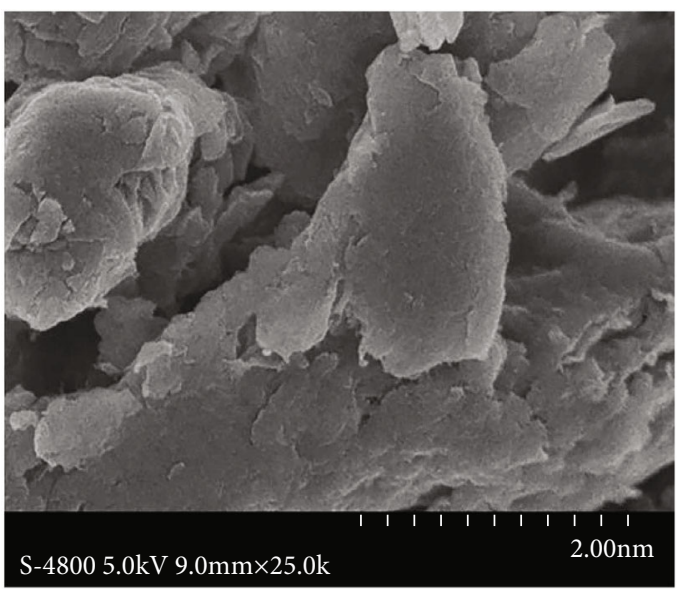

(b)

Figure 2: SEM images of (a) CNTs and (b) GO.

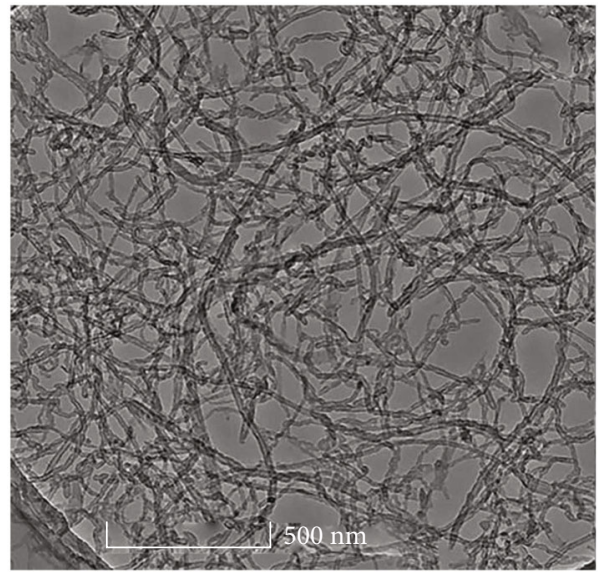

(a)

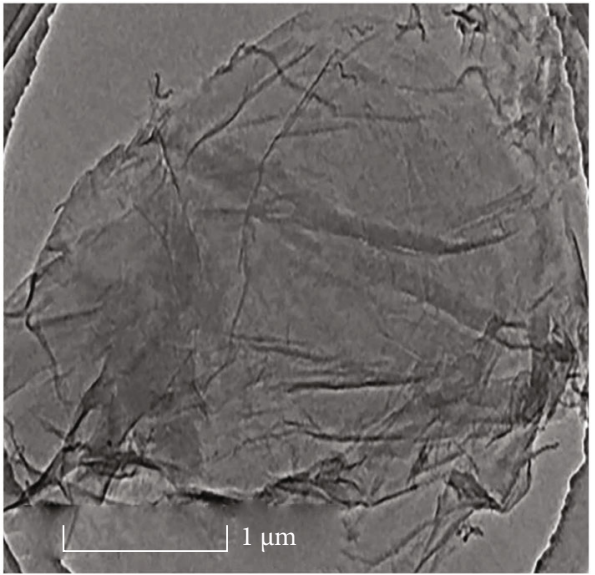

(b)

Figure 3: TEM images of (a) CNTs and (b) GO. 


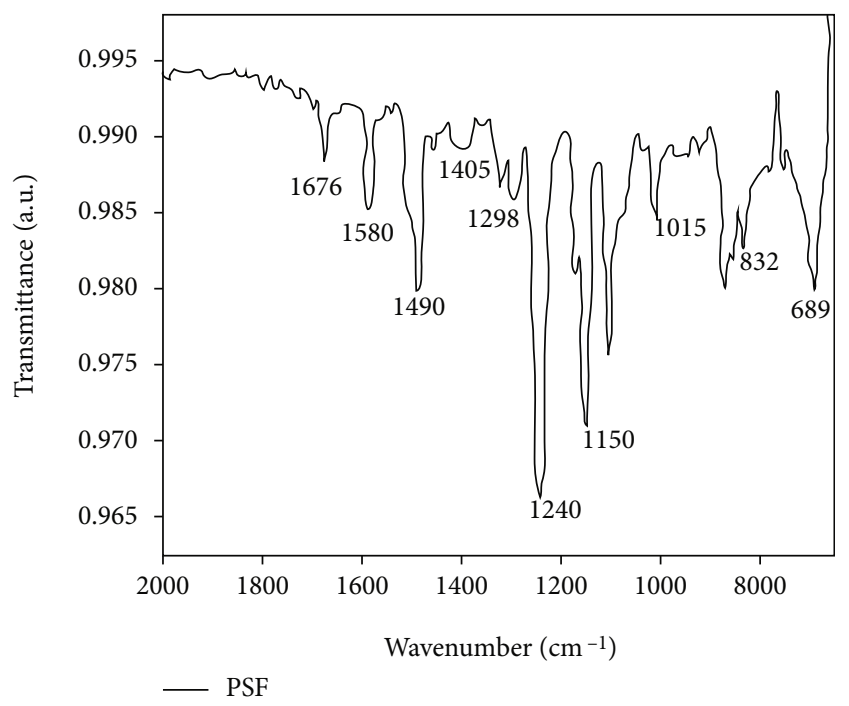

(a)

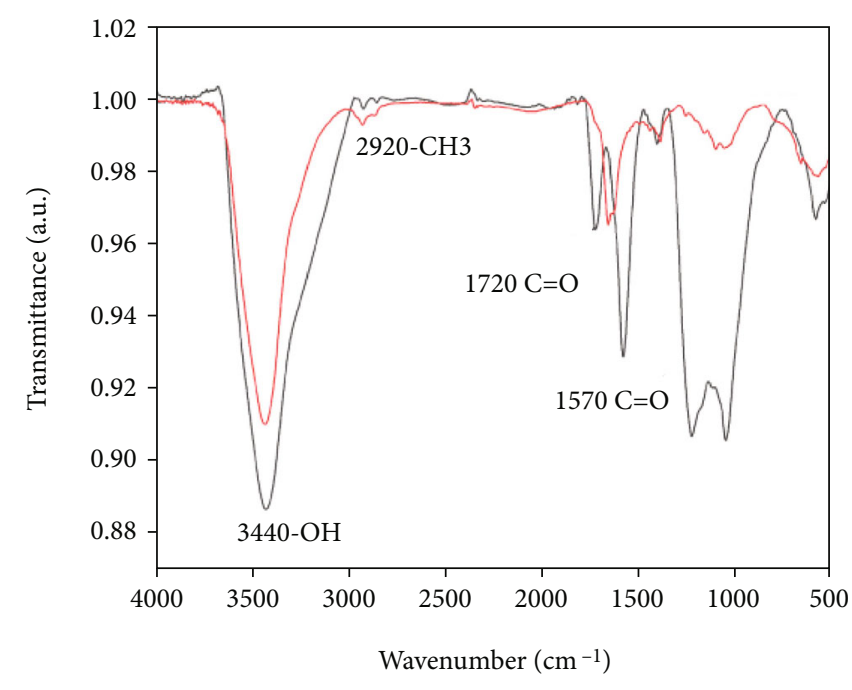

- GO

(b)

FIgUre 4: FTIR images of (a) PSF and (b) CNTs and GO.

membranes under external pressure, the membrane separation method can be used to separate gas. Compared with other methods, the membrane method has many advantages, such as being economical and environmentally friendly, having low energy consumption, and easy modification. Therefore, the method has promising research prospects in the field of gas separation at present [6-10]. Based on differences in materials, gas separation membrane can be divided into various types, such as inorganic, organic, and mixed matrix. The inorganic membrane has zeolite, molecular sieve, graphene, metalorganic framework, and other inorganic materials with porous structure as the main body [11-14], and it conducts gas permeation and separation based on the pores of the materials. The membrane has high temperature resistance, stable chemical properties, high strength, and adjustable pore size. Lin et al. [15] prepared zeolite/carbon hybrid membranes and studied the permeability and ideal separation performance of different gases. Among these gases, the permeability coefficients of $\mathrm{CO}_{2}$ and $\mathrm{N}_{2}$ were 564 Barrer and 15 Barrer, respectively. The ideal separation coefficients of $\mathrm{CO}_{2} / \mathrm{N}_{2}$ could reach 37.7 (the diameter of ZSM-5 doped particle was $0.2 \mu \mathrm{m}$ ). Japip et al. [16] prepared 6FDA-Durene/ZIF-71 mixed matrix membrane and studied the effect of ZIF-71 of different particle sizes on the selectivity of gas. The selectivity of $\mathrm{O}_{2} / \mathrm{N}_{2}, \mathrm{CO}_{2} / \mathrm{N}_{2}$, and $\mathrm{CO}_{2} / \mathrm{CH}_{4}$ could reach $3.35,14.7$, and 17.0, respectively. Although molecular sieve has shown excellent characteristics in the field of gas separation, its application in practical production is limited due to the complex preparation process, the preparation process is easy to cause adverse effects on the environment, and it is of high cost.

In 1991, a new type of carbon material, a 1D tube known as a carbon nanotube, was founded by Dr. Iijima from Japan NEC Company. The CNT comprises a curl of graphene around a central axis [17]. In recent years, with the continuous research on CNTs, CNTs have been shown to possess good

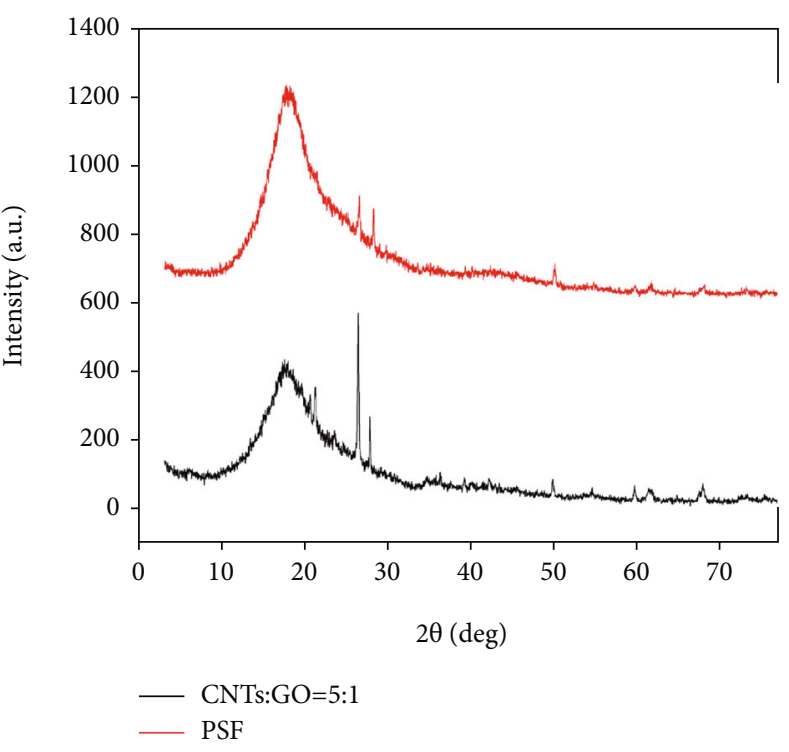

FIGURE 5: XRD of PSF and PSF/GO/CNT MMMs.

thermal, electrical, optical, and mechanical properties [18]. With good heat conduction and conductivity, high hardness, high strength, large specific surface area, and high lengthdiameter aspect ratio [19], the nanotube can be compounded with polymer materials and inorganic nonmetallic materials and applied to many fields, including water treatment, gas separation, energy, and sensors. The relevant research is also found in gas separation.

Graphene, as a 2D, is composed of carbon atoms by $\mathrm{sp}^{2}$ hybridization, which is a compact lamellar structure in the form of a six-membered ring. It has a large specific surface area due to its unique $2 \mathrm{D}$ plane structure [20]. At the same 
time, the layers are connected by van der Waals force. With a small acting force, the relative movement among layers is easily produced [21]. GO is obtained by the oxidation of graphene. The lamellar structure is loaded with oxygencontaining groups, thereby increasing the gap among layers and making obtaining graphene with fewer layers easier [22, 23 . At the same time, owing to the introduction of oxygencontaining groups, the hydrophilicity of graphene is increased, thereby providing a site for further modification. With these excellent properties, GO has also been applied to gas separation [24]. Li et al. [25] prepared a GO film with a thickness of only $1.8 \mathrm{~nm}$ and explored gas separation performance. The selectivity of $\mathrm{H}_{2} / \mathrm{CO}_{2}$ could reach 3400 . Yang et al. [26] prepared the separation film for separating $\mathrm{H}_{2}$ and $\mathrm{CO}_{2}$ by using self-assembly technology. Its selectivity exceeded 300 . Koenig et al. [27] prepared a GO film for separating $\mathrm{H}_{2}$ and $\mathrm{CH}_{4}$ by using oxygen etching. Its selectivity could reach $10^{4}$. Kemal et al. [28] prepared a separation membrane with a bimolecular graphene layer. Its selectivity for $\mathrm{H}_{2} / \mathrm{CO}_{2}$ was 4.6. Kim et al. [24] deposited GO on the surface of the substrate by chemical vapor deposition and prepared the separation membrane. Its selectivity for $\mathrm{O}_{2} / \mathrm{N}_{2}$ could reach 6 , and the selectivity of $\mathrm{GO} /$ polyethersulfone composite film prepared by the spin coating method for $\mathrm{CO}_{2} / \mathrm{N}_{2}$ could reach 20. Shen et al. [29] prepared a GO-PEBA membrane, and its selectivity for $\mathrm{CO}_{2} / \mathrm{N}_{2}$ could reach 91 . The selectivity of the ZIF-8@GO inorganic separation membrane prepared by Huang et al. using the layer-by-layer method for $\mathrm{H}_{2} / \mathrm{N}_{2}$ could also reach 91 [30]. Athanasekou et al. [31] prepared a GO membrane by using AAO as the base material and studied the membrane's permeability to gas and water. The prepared membrane has good permeability to water and helium but relatively weak permeability to $\mathrm{N}_{2}$.

Inorganic separation membranes commonly used in gas separation have some disadvantages, such as high preparation cost, high brittleness, and complex preparation process. Compared with inorganic separation membrane, organic separation membrane, for example, polyethersulfone membrane, has the advantages of low preparation cost, simple film-forming process, strong flexibility, and so on, but also has the disadvantage of higher separation efficiency than inorganic membrane, which restricts the development of pure organic membrane. Therefore, the mixed matrix membrane combining organic and inorganic materials has become a research hotspot in recent years. Orooji et al. [32] reported a kind of polyethersulfone membrane with nanostructured mesoporous carbon composited that showed adding the mesoporous carbon nanoparticles improved the pure water flux. Orooji's team using polyethersulfone prepared ultrafiltration membrane, and results showed that adding vermiculite can improve mixed matrix membrane flux to pure polyethersulfone membrane [33].

In this paper, the mixed matrix membrane (MMM) is prepared by taking PSF, GO, and CNTs as raw materials. The prepared materials are characterized by scanning electron microscopy (SEM), transmission electron microscopy (TEM), $\mathrm{X}$-ray diffraction (XRD), Fourier infrared spectroscopy (FTIR), and Raman spectroscopy. The permeability of $\mathrm{CO}_{2}$ and $\mathrm{N}_{2}$ with a single component is tested by using laboratory self-assembly devices, and the ideal separation coefficient is calculated.

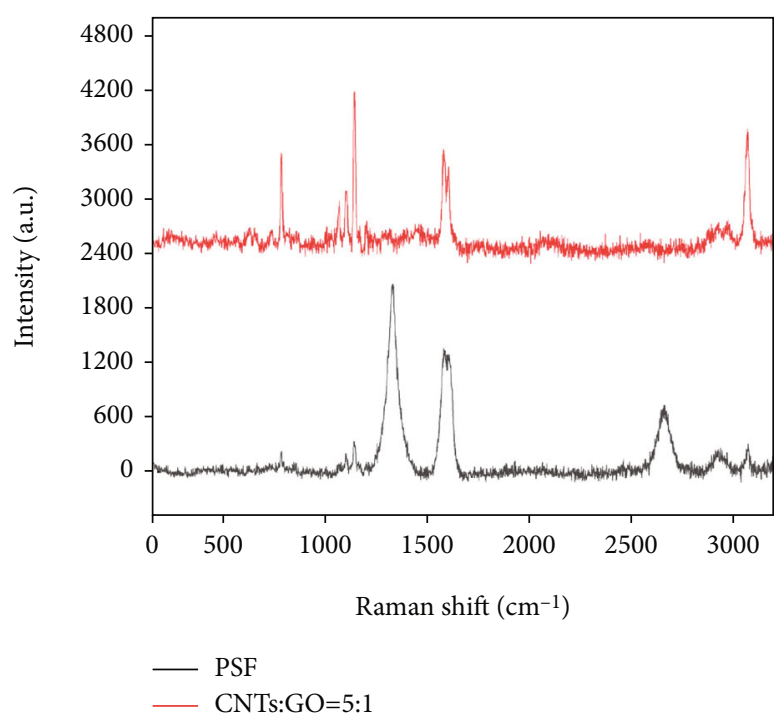

FIGURE 6: Raman spectroscopy images of PSF and PSF/GO/CNTs.

\section{Experiments and Methods}

2.1. Materials and Reagents. SWCNTs were purchased from Shenzhen Nanotech Port Co., Ltd. (China), which was treated with mixed acids (volume ratio $\mathrm{H}_{2} \mathrm{SO}_{4}: \mathrm{HNO}_{3}=3: 1$ ). Natural flake graphite (12,000 mesh) was purchased from Qingdao Tianshengda Graphite Co., Ltd. (China). $\mathrm{H}_{2} \mathrm{SO}_{4}, \mathrm{HNO}_{3}$, and $\mathrm{KMnO}_{4}$ were purchased from Big Alum Chemical Reagent Factory (China). Ultrapure water was used for the preparation of solution. All of the chemicals were of analytical grade $(>99.7 \%)$ without further purification. $\mathrm{N}_{2}, \mathrm{CO}_{2}$, and mixed gas $\left(\mathrm{N}_{2}: 60 \%, \mathrm{CO}_{2}: 20 \%\right.$, and $\left.\mathrm{CO}: 20 \%\right)$ were purchased from Shanghai Tonghui Special Gas Co., Ltd. Polysulfone (PSF) was purchased from Aldin Co., Ltd.

\subsection{Preparation of $M M M s$}

2.2.1. Preparation of GO. With natural flake graphite as a raw material, GO is prepared by the existing improved Hummer method. Graphite is first treated with mixed acid, and then, GO is prepared by three stages of reaction at low, medium, and high temperature. Details may be found in the articles of the research team [34].

\subsubsection{Preparation of PSF Mixed Matrix Membrane. Different} qualities of PSF $(0.5,0.4985,0.497$, and $0.4945 \mathrm{~g})$ are weighed and placed in a flat-bottomed flask with $10 \mathrm{~mL}$ DMF. The mixture is stirred magnetically at $80^{\circ} \mathrm{C}$. As a result, PSF is completely dissolved in DMF. Then, GO and CNTs are added into $5 \mathrm{~mL}$ DMF. Ultrasound is conducted for $3 \mathrm{~h}$ to ensure complete dispersal. Under ultrasonic conditions, the completely dispersed GO/CNTs are added into the PSF solution slowly, and the ultrasound is conducted continuously for $4 \mathrm{~h}$ to ensure that GO/CNTs are completely dispersed in the PSF solution. The solution is heated at $80^{\circ} \mathrm{C}$ for $10 \mathrm{~min}$ and then poured into a dish preheated for $30 \mathrm{~min}$ in a vacuum drying oven at $80^{\circ} \mathrm{C}$ to heat and dry for $12 \mathrm{~h}$. As a result, the mixed matrix membranes mixed 


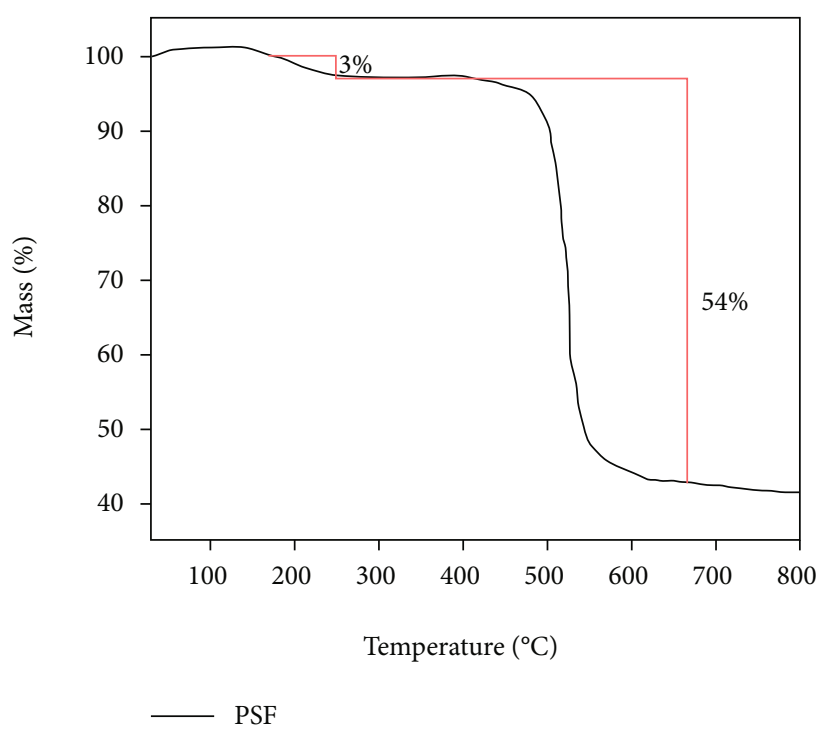

(a)

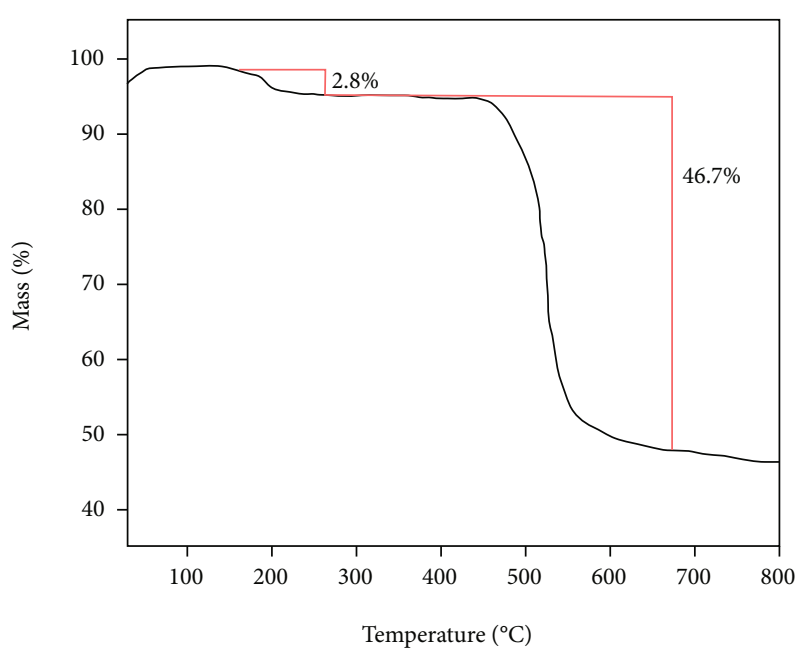

- CNTs:GO=5:1

(b)

Figure 7: TGA of (a) PSF and (b) PSF/GO/CNTs.

with different contents of CNTs and GO are obtained. The ratio of CNT and GO is $0,2: 1,5: 1$, and $10: 1$, respectively.

2.3. Experimental Apparatus for Gas Separation. Taking $\mathrm{CO}_{2}$ and $\mathrm{N}_{2}$ as single-component gases, a gas permeation test is conducted for the mixed matrix membrane under different intake pressures $\left(\mathrm{CO}_{2}\right.$ intake pressure is $0.1,0.12,0.14$, $0.16,0.18$, and $0.2 \mathrm{MPa} ; \mathrm{N}_{2}$ intake pressure is $0.1,0.15$, and $0.2 \mathrm{MPa}$ ). The permeability coefficient is obtained by data calculation to characterize the gas permeability of the prepared membrane. The permeability coefficient is calculated based on the following formula (1) [35]:

$$
P=\frac{Q 1}{A \Delta p}=\frac{273 \times 10^{10}}{760} \frac{V l}{A T\left[P_{0} \times 76 / 14.7\right]} \frac{d_{p}}{d_{t}},
$$

where $Q$ represents the permeability flux of gas; $\Delta p$ is the gas pressure difference on both sides of the membrane, $\mathrm{mm} / \mathrm{Hg}$; $P$ is the permeability coefficient, Barrer; $V\left(\mathrm{~cm}^{3}\right)$ is the volume of the permeability chamber; $l(\mathrm{~cm})$ is the thickness of the film; $A\left(\mathrm{~cm}^{2}\right)$ is the effective area of the membrane; $T$ $(\mathrm{K})$ is the experimental temperature; $P_{0}$ refers to the initial intake pressure at the beginning of the experiment in the unit psia; $d_{p} / d_{t}$ refers to the pressure increment over time in the infiltration chamber.

The ideal separation coefficient is calculated based on the following formula (2):

$$
\partial_{i}=\frac{P_{A}}{P_{B}}
$$

where $\partial_{i}$ represents the ideal separation coefficient; $P_{A}$ and $P_{B}$ represent permeability coefficient of different gas $A$ and $B$, respectively. The experimental devices and methods are shown in Figure 1.
2.4. Characterization Techniques. Fourier transform infrared spectroscopy (FTIR, type Nicotct 5700) was used to characterize the related groups of PSF and the treated carbon materials. Scanning electron microscopy (SEM, type JEM-6700F, operating at $0.5-30 \mathrm{kV}$ ) and transmission electron microscopy (TEM, type JEM-2010, operating at $200 \mathrm{kV}$ ) were used to analyze the morphology of the prepared GO and purified CNTs. X-ray diffraction (XRD, type D8/AXS, operating at $60 \mathrm{kV}$ ) and Raman spectroscopy (type inVia) were used to relatively characterize the existence of GO and CNTs in the mixed matrix membrane. TGA (type NETZSCHSTA$449 \mathrm{~F} 3$ ) is used to analyze the thermal stability of prepared gas separation membranes under the $\mathrm{N}_{2}$ atmosphere from room temperature to $800^{\circ} \mathrm{C}$ at a heating rate of $20^{\circ} \mathrm{C} / \mathrm{min}$.

\section{Results and Discussion}

3.1. SEM. Figure 2 shows the surface morphology of CNTs and GO composites. Figure 2(a) clearly shows that the purified CNTs are scattered without obvious agglomeration, and the length of CNTs was shortened. Figure 2(b) shows the surface morphology of prepared GO, with obvious lamellar structure and fewer stacking layers, thereby indicating that GO is successfully prepared.

3.2. TEM. Figure 3(a) clearly shows hollow tubular CNTs did not have impurities and obvious agglomeration and showed good dispersion. Figure 3(b) shows the lamellar structure of GO has fewer stacking layers with a good separation effect.

3.3. FTIR. In Figure 4(a), a small peak occurs at $2965 \mathrm{~cm}^{-1}$, which may be the stretching vibration peak of $\mathrm{C}-\mathrm{H}$ due to the wide peak shape. The peak at $1676 \mathrm{~cm}^{-1}$ is clear, and no other peaks are nearby, thereby indicating that it is the $\mathrm{C}=\mathrm{O}$ stretching vibration peak of $-\mathrm{COOH}$, whereas the peaks at 1580 and $1490 \mathrm{~cm}^{-1}$ are the characteristic absorption peaks of the benzene ring in the PSF structure $[36,37]$. The peak 


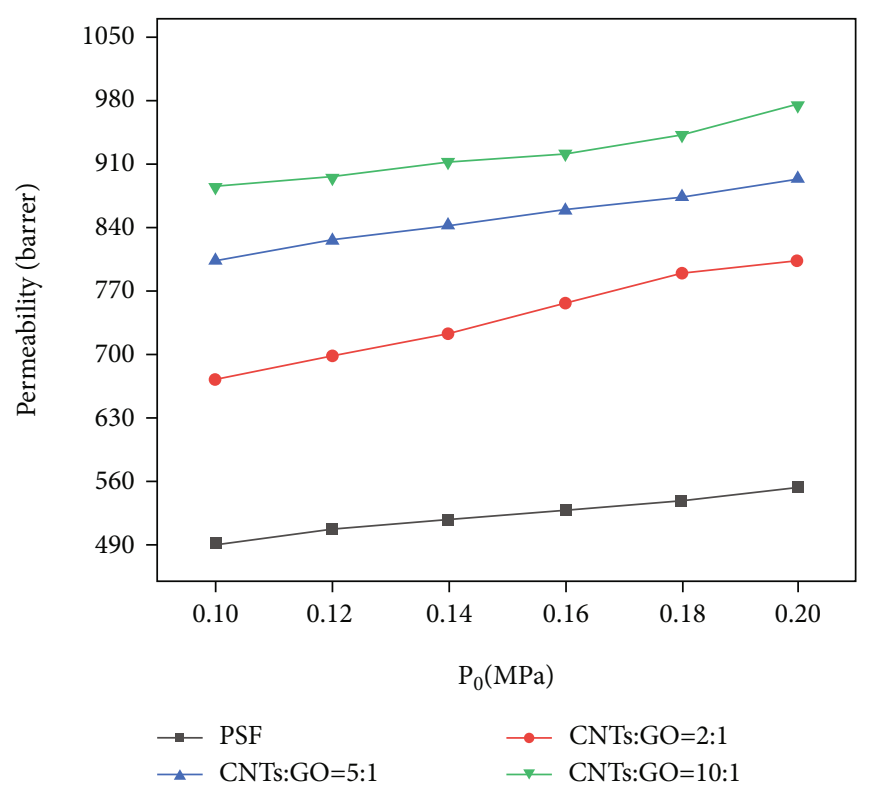

(a)

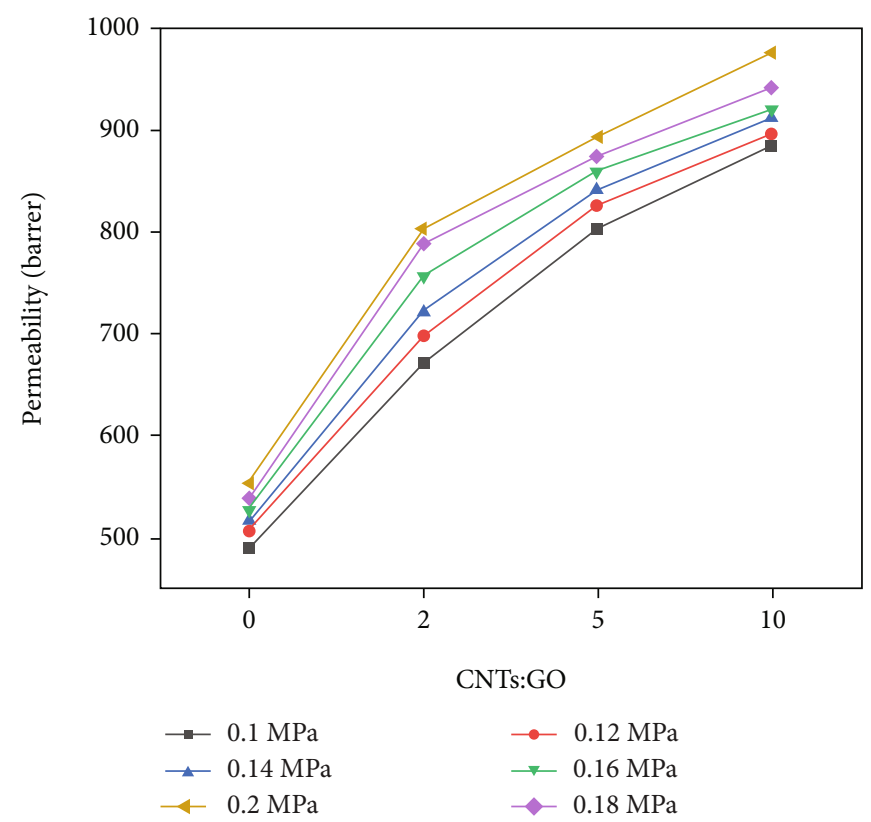

(b)

Figure 8: (a) $\mathrm{CO}_{2}$ permeability of PSF and PSF/GO/CNTs and (b) with different inlet pressure.

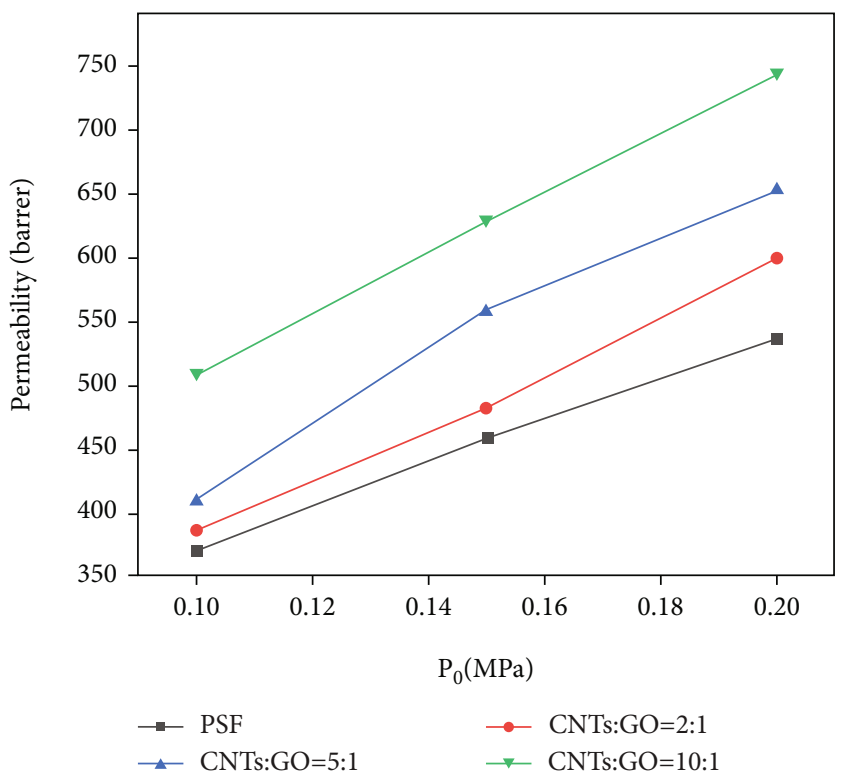

(a)

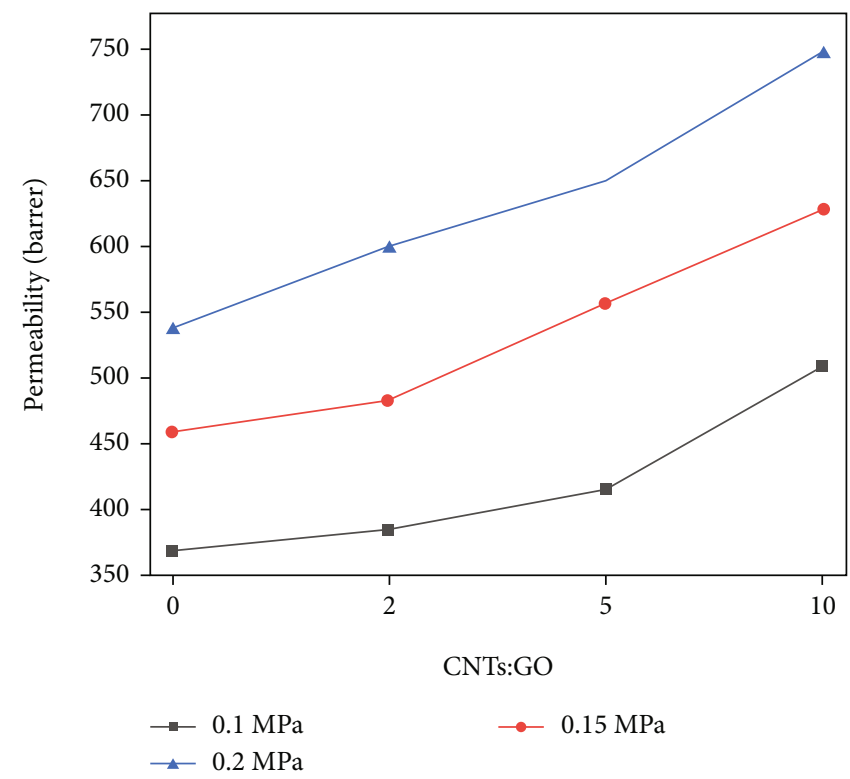

(b)

Figure 9: (a) $\mathrm{N}_{2}$ permeability of PSF and PSF/GO/CNTs and (b) $\mathrm{N}_{2}$ permeability of PSF/GO/CNTs at different inlet pressure.

at 832 and $689 \mathrm{~cm}^{-1}$ also proves the existence of the benzene ring. The peak at $1405 \mathrm{~cm}^{-1}$ corresponds to the bending vibration peak of the $\mathrm{O}-\mathrm{H}$ bond. The peaks at 1298 and $1150 \mathrm{~cm}^{-1}$ should be the absorption peak of the sulfone group $(\mathrm{O}=\mathrm{S}=\mathrm{O})$ in the PSF structure. The peak at $1240 \mathrm{~cm}^{-1}$ is the strongest in the spectrum, thereby indicating that it stretches the vibration peak of the C-O group in the PSF structure. The peak at $1015 \mathrm{~cm}^{-1}$ may be the absorption peak of the C-
O-C group. Figure 3(b) shows the FTIR spectrum of GO and $\mathrm{CNTs}$. The GO and CNT spectra show a strong $-\mathrm{OH}$ vibration peak at $3440 \mathrm{~cm}^{-1}$, thereby indicating that the surface of the prepared GO and the surface of CNTs treated by mixed acid are loaded with -OH. The peak at $2920 \mathrm{~cm}^{-1}$ is a vibration peak of $-\mathrm{CH}_{3}$, and that at $1720 \mathrm{~cm}^{-1}$, it is a $\mathrm{C}=\mathrm{O}$ vibration peak, indicating the introduction of carboxyl, and that at $1570 \mathrm{~cm}^{-}$ ${ }^{1}$, it is a $\mathrm{C}=\mathrm{C}$ vibration peak. Owing to the existence of 


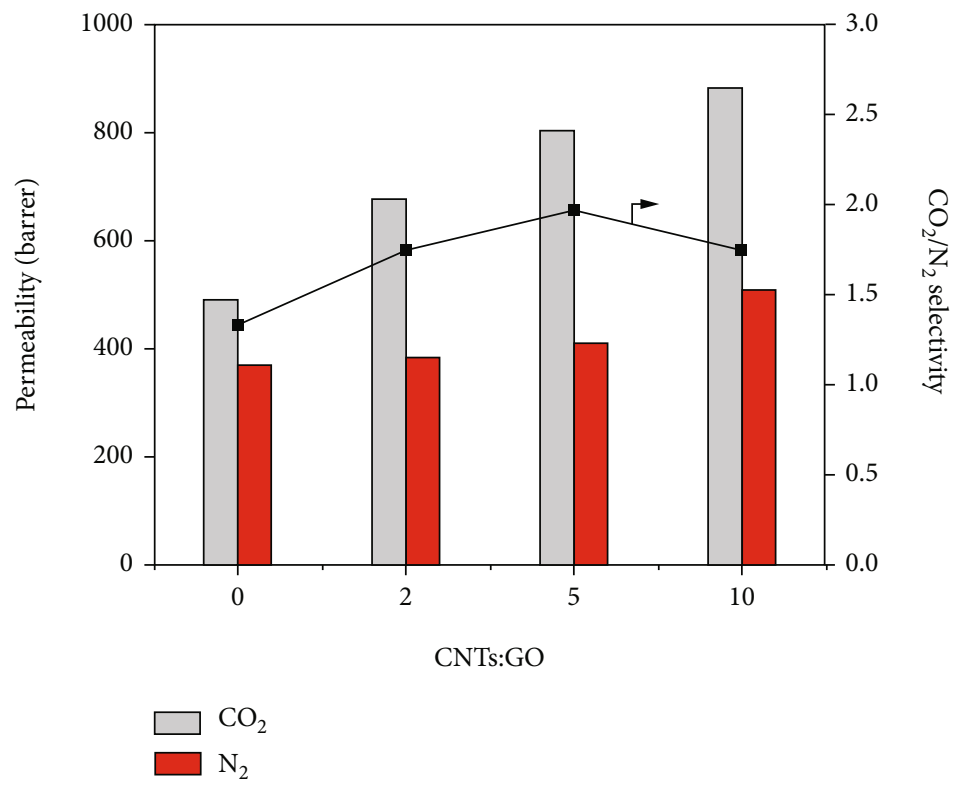

Figure 10: Permeability and selectivity of PSF/GO/CNTs.

hydroxyl and carboxyl groups, GO was successfully prepared from graphite, and CNTs were successfully purified by mixed acid treatment [38-40].

3.4. XRD. Figure 5 shows that the pure PSF membrane has a very strong peak at $18^{\circ}$. This peak is the crystal plane diffraction of PSF, thereby indicating that the pure PSF has a certain degree of crystallinity. After the doping of CNT and $\mathrm{GO}$, this diffraction peak obviously remains, indicating that the doping did not destroy the structure of the polymer itself. Compared with the pure PSF, the diffraction peak moved towards a low angle (blue shift), indicating that the addition of CNTs and GO increased the spacing of the molecular chain, although the peak strength decreased. The addition of CNTs and GO increased the degree of freedom and free volume of the polymer chain, decreased the crystallization behavior caused by the aggregation and stacking of molecular chains, and lowered the crystallinity of the mixed matrix membrane itself $[41,42]$. Compared with the pure PSF membrane, because of the addition of CNTs and GO, two strong peaks existed near $26.8^{\circ}$. These peaks occurred in the pure PSF and mixed matrix membranes, and the peak in the mixed matrix membrane had higher strength. At the same time, CNTs have characteristic peaks here, thereby proving the existence of CNTs in the mixed matrix membrane $[34,43]$.

3.5. Raman Spectroscopy. CNTs and GO in the prepared mixed matrix membrane can be analyzed by Raman spectroscopy. Figure 6 shows that the strong peaks occurred at $1147 \mathrm{~cm}^{-1}, 1583 \mathrm{~cm}^{-1}$, and $3050 \mathrm{~cm}^{-1}$ correspond to PSF, respectively, which is basically consistent with previous reports. The comparison between PSF and PSF/GO/CNT membrane shows that while oxygen-containing groups are introduced in the preparation of GO and CNTs and chemical treatment, some structural defects and $\mathrm{sp}^{2}$ hybridization of $\mathrm{C}$ atom are caused. Thus, a $\mathrm{D}$ peak is present at $1365 \mathrm{~cm}^{-1}$, and a $G$ peak is at $1660 \mathrm{~cm}^{-1}$. However, a $2 \mathrm{D}$ peak of $\mathrm{GO}$ and CNTs is at $2600 \mathrm{~cm}^{-1}$. The appearance of $\mathrm{D}, \mathrm{G}$, and $2 \mathrm{D}$ peaks of GO and CNTs proved the dispersion of GO and CNTs in PSF [43-47].

3.6. TGA. Figure 7 shows that there are two stages of mass reduction in pure PSF membrane and PSF/GO/CNTs. The first stage of weightlessness occurs at $250^{\circ} \mathrm{C}$, which is mainly caused by the fracture of polymer molecular chain at this temperature. With further increase of temperature, the second stage of weightlessness appears around $520^{\circ} \mathrm{C}$. It indicates that the polymer is decomposed and carbonized at this temperature. And the molecules are removed in the form of small molecular compounds, so that the mass of membrane is significantly reduced. The similar curves also show that the second stage of weightlessness of the mixed matrix membrane is obviously smaller than that of pure PSF membrane, and the remaining mass is also more than that of the PSF membrane. Thus, the weight loss near $520^{\circ} \mathrm{C}$ is due to the carbonization and decomposition of the membrane.

3.7. The Permeability of $\mathrm{CO}_{2}$ with Different Pressure. Figure 8 shows that intake pressure and addition of CNTs/GO have a certain influence on the permeability coefficient of $\mathrm{CO}_{2}$. The increase in the permeability coefficient can be significantly improved by increasing the intake pressure or the addition of CNTs. Increasing the content of CNTs can improve the permeability of $\mathrm{CO}_{2}$ more obviously than increasing the intake pressure. At $0.1 \mathrm{MPa}$, the permeability coefficient of $\mathrm{CO}_{2}$ is increased by 303 Barrer, from 491 Barrer (pure PSF membrane) to 884 Barrer (CNT : GO =10:1); at $0.2 \mathrm{MPa}$, it is increased by 422 Barrer from 553 Barrer (pure PSF membrane) to 975 Barrer (CNTs : $\mathrm{GO}=10: 1$ ). If only intake pressure is changed from $0.1 \mathrm{MPa}$ to $0.2 \mathrm{MPa}$, and the ratio of CNTs to GO remains unchanged, then permeability 
TABLE 1: $\mathrm{CO}_{2}$ permeability factor of different PSF MMMs.

\begin{tabular}{lcccc}
\hline Fillers & $\begin{array}{c}\text { Polymer } \\
\text { matrix }\end{array}$ & Filler (Wt.\%) & $\begin{array}{c}\text { PF } \\
\left(\mathrm{CO}_{2, \mathrm{GPU}}\right)\end{array}$ & Ref. \\
\hline CNTs & PSF & 10 & 1.33 & {$[48]$} \\
& PSF & 0.5 & 7.14 & {$[49]$} \\
CNF & PSF & 1 & 6.86 & {$[50]$} \\
& PSF & 0.25 & 1.14 & {$[51]$} \\
GO & PSF & 30 & 1.43 & {$[52,53]$} \\
CNTs/ & PSF & CNTs 1; GO & 7.5 & This \\
GO & & 0.1 & & paper \\
\hline
\end{tabular}

coefficient of $\mathrm{CO}_{2}$ is increased by 131 Barrer at most. The permeation of $\mathrm{CO}_{2}$ through this membrane is positively correlated with the pore content of the membrane. Given that CNTs are hollow tubular structures, the increase of CNTs to $\mathrm{GO}$ ratio provides more channels for $\mathrm{CO}_{2}$ diffusion and permeation, thereby improving the surface area of the membrane and promoting the probability of $\mathrm{CO}_{2}$ diffusion through the surface. As a result, the permeability coefficient of $\mathrm{CO}_{2}$ is significantly increased.

3.8. The Permeability of $\mathrm{N}_{2}$ with Different Pressure. Figure 9 shows the influence of different intake pressures and the ratio of CNTs to GO on the permeability coefficient of $\mathrm{N}_{2}$. The same as $\mathrm{CO}_{2}$, the permeability coefficient of $\mathrm{N}_{2}$ can be improved by increasing the intake pressure and the ratio of CNTs to GO. At $0.1 \mathrm{MPa}$, by changing the addition of CNTs, the permeability coefficient of $\mathrm{N}_{2}$ is increased by 123 Barrer from 385 Barrer to 508 Barrer. At $0.2 \mathrm{MPa}$, the coefficient is increased by 209 Barrer from 536 Barrer to 745 Barrer. If the ratio of CNTs to GO remains unchanged, and the intake pressure is changed from $0.1 \mathrm{MPa}$ to $0.2 \mathrm{MPa}$, then the permeability of $\mathrm{N}_{2}$ is increased by 237 Barrer at most. Increasing of the intake pressure more obviously improved $\mathrm{N}_{2}$ permeability than that of the ratio of CNTs to GO. However, after increasing the ratio of CNTs to GO, the permeability coefficient of $\mathrm{N}_{2}$ is significantly increased, which is significantly different from the permeability of $\mathrm{CO}_{2}$. The permeation mechanism of $\mathrm{N}_{2}$ is different from that of $\mathrm{CO}_{2}$ in terms of passing through this mixed matrix membrane. Given that the molecular dynamic diameter of $\mathrm{N}_{2}(0.364 \mathrm{~nm})$ is larger than that of $\mathrm{CO}_{2}(0.33 \mathrm{~nm}), \mathrm{N}_{2}$ may focus on Knudsen diffusion. Its diffusion rate is directly proportional to the pressure difference on both sides of the membrane. Owing to the increase in the ratio of CNTs to GO, the collision probability occurs between $\mathrm{N}_{2}$ molecules and pore wall. Therefore, the collision between gas molecules and pore wall is much more than that between molecules, thereby improving the permeability coefficient of $\mathrm{N}_{2}$.

3.9. The Separation Performance of $\mathrm{CO}_{2}$ and $\mathrm{N}_{2}$. Figure 10 shows that at $0.1 \mathrm{MPa}$, the permeability coefficient of $\mathrm{CO}_{2}$ is obviously higher than that of $\mathrm{N}_{2}$. With increasing ratio of CNTs to $\mathrm{GO}$, the permeability coefficient of $\mathrm{CO}_{2}$ increased more obviously than that of $\mathrm{N}_{2}$. However, when the ratio of CNTs to GO increased from $5: 1$ to $10: 1$, the increase in the permeability coefficient of $\mathrm{N}_{2}$ was more than that of $\mathrm{CO}_{2}$. The number of pores also played an important role in improving the permeability of $\mathrm{N}_{2}$. Therefore, ratio of CNTs to GO is less than $5: 1$, and the effect of pore numbers on permeability for $\mathrm{CO}_{2}$ was more obvious. With further increase in the ratio, the effect of pores on $\mathrm{N}_{2}$ became more obvious. At $0.1 \mathrm{MPa}$, when the ratio of CNTs to GO was $5: 1$, the ideal permeability coefficient of $\mathrm{CO}_{2} / \mathrm{N}_{2}$ was 1.94 , whereas the ideal permeability coefficient of PSF membrane was 1.46. The addition of CNTs and GO can effectively improve the selectivity of the membrane.

The comparison of this paper with the published articles is shown in Table 1. The separation membrane prepared by this research team greatly improved the permeability of $\mathrm{CO}_{2}$ and $\mathrm{N}_{2}$. GO and CNTs can effectively improve the permeability of gas, thereby providing a direction and possibility for exceeding trade-off upper limits.

\section{Conclusions}

In this paper, the improved Hummer method is used to prepare GO from natural flake graphite, and CNTs are treated by the mixed acid method. Taking the two carbon materials as additives and PSF as the substrate, the mixed matrix membrane was prepared. Compared with the pure PSF membrane, the gas permeability was greatly improved after adding CNTs and GO. At the same intake pressure, the difference in the permeability of $\mathrm{CO}_{2}$ between PSF and PSF/GO/CNTs can be 422 Barrer at $0.2 \mathrm{MPa}$ at most, and that of the permeability of $\mathrm{N}_{2}$ can be 209 Barrer. The permeability of CNTs for $\mathrm{CO}_{2}$ was improved more significantly than that of $\mathrm{N}_{2}$. Increasing the intake pressure was more effective for improving the permeability of $\mathrm{N}_{2}$. At $0.1 \mathrm{MPa}$, when the ratio of CNTs to GO was $5: 1$, the ideal permeability coefficient of $\mathrm{CO}_{2} / \mathrm{N}_{2}$ was 1.94, whereas the ideal permeability coefficient of PSF membrane was 1.46 .

\section{Data Availability}

All the data supporting the results are shown in the paper and can be applicable from the corresponding author.

\section{Conflicts of Interest}

The authors declare that there are no competing interests regarding the publication of this paper.

\section{Acknowledgments}

The authors are grateful for the financial support from the National Natural Science Foundation of China (51764039); Basic Public Welfare Research Project of Zhejiang Province (LQ19E040006); State Key Laboratory of Advanced Processing and Recycling of Non-Ferrous Metals, Lanzhou University of Technology (SKLAB02019013); Doctor Research Foundation of Lanzhou University of Technology (061702); and Project of Hongliu support discipline of Lanzhou University of Technology. 


\section{References}

[1] C. Karaman, O. Karaman, N. Atar, and M. L. Yola, "Tailoring of cobalt phosphide anchored nitrogen and sulfur co-doped three dimensional graphene hybrid: boosted electrocatalytic performance towards hydrogen evolution reaction," Electrochimica Acta, vol. 380, article 138262, 2021.

[2] M. S. Lashkenari, M. Ghorbani, N. Silakhori, and H. KarimiMaleh, "Enhanced electrochemical performance and stability of $\mathrm{Pt} / \mathrm{Ni}$ electrocatalyst supported on $\mathrm{SiO}_{2}$-PANI nanocomposite: a combined experimental and theoretical study," Materials Chemistry and Physics, vol. 262, p. 124290, 2021.

[3] C. Karaman, E. Bayram, O. Karaman, and Z. Aktaş, "Preparation of high surface area nitrogen doped graphene for the assessment of morphologic properties and nitrogen content impacts on supercapacitors," Journal of Electroanalytical Chemistry, vol. 868, article 114197, 2020.

[4] G. L. Zhuang, H. H. Tseng, and M.-Y. Wey, "Facile synthesis of $\mathrm{CO}_{2}$-selective membrane derived from butyl reclaimed rubber (BRR) for efficient $\mathrm{CO}_{2}$ separation," Journal of CO2 Utilization, vol. 25, pp. 226-234, 2018.

[5] Á. A. Ramírez-Santos, M. Bozorg, B. Addis, C. Castel, and E. Favre, "Optimization of multistage membrane gas separation processes. Example of application to $\mathrm{CO}_{2}$ capture from blast furnace gas," Journal of Membrane Science, vol. 566, pp. 346-366, 2018.

[6] A. S. Kovvali, H. Chen, and K. K. Sirkar, "Dendrimer membranes: $\mathrm{CO}_{2}$-selective molecular gate," Journal of the American Oil Chemists Society, vol. 122, no. 31, pp. 7594-7595, 2000.

[7] R. W. Baker, "Future directions of membrane gas-separation technology," Industrial \& Engineering Research, vol. 41, no. 6, pp. 1393-1411, 2002.

[8] W. J. Koros and G. K. Fleming, "Membrane-based gas separation," Journal of Membrane Science, vol. 83, no. 1, pp. 1-80, 1993.

[9] A. S. Kovvali and K. K. Sirkar, "Dendrimer liquid membranes: $\mathrm{CO}_{2}$ separation from gas mixtures," Industrial \& Engineering Research, vol. 40, no. 11, pp. 2502-2511, 2001.

[10] W. J. Lau and P. D. Antonia, "Membrane separation," Chemical Engineering \& Technology, vol. 41, no. 2, pp. 210-210, 2018.

[11] S. J. Ahn, A. Takagaki, T. Sugawara, R. Kikuchi, and S. T. Oyama, "Permeation properties of silica-zirconia composite membranes supported on porous alumina substrates," Journal of Membrane Science, vol. 526, pp. 409-416, 2017.

[12] A. M. Marti, W. Wickramanayake, G. Dahe et al., "Continuous flow processing of ZIF- 8 membranes on polymeric porous hollow fiber supports for CO2 capture," ACS Applied Materials \& Interfaces, vol. 9, no. 7, pp. 5678-5682, 2017.

[13] H. H. Tseng, C. T. Wang, G. L. Zhuang, P. Uchytil, J. Reznickova, and K. Setnickova, "Enhanced $\mathrm{H}_{2} / \mathrm{CH}_{4}$ and $\mathrm{H}_{2} / \mathrm{CO}_{2}$ separation by carbon molecular sieve membrane coated on titania modified alumina support: effects of $\mathrm{TiO}_{2}$ intermediate layer preparation variables on interfacial adhesion," Journal of Membrane Science, vol. 510, pp. 391-404, 2016.

[14] M. Kanezashi, T. Matsutani, T. Wakihara et al., "Tailoring the subnano silica structure via fluorine doping for development of highly permeable $\mathrm{CO}_{2}$ separation membranes," ChemNanoMAT, vol. 2, no. 4, pp. 264-267, 2016.

[15] L. Li, C. Wang, N. Wang, Y. Cao, and T. Wang, "The preparation and gas separation properties of zeolite/carbon hybrid membranes," Journal of Materials Science, vol. 50, no. 6, pp. 2561-2570, 2015.

[16] S. Japip, Y. C. Xiao, and T. S. Chung, "Particle-size effects on gas transport properties of 6FDA-Durene/ZIF-71 mixed matrix membranes," Industrial \& Engineering Chemistry Research, vol. 55, pp. 5907-5917, 2016.

[17] S. Iijima, "Helical microtubules of graphitic carbon," Nature, vol. 354, no. 6348, pp. 56-58, 1991.

[18] X. You, X. M. Sun, and H. S. Peng, "Aligned carbon nanotube fiber for energy conversion and storage," Materials China, vol. 33, pp. 449-457, 2014.

[19] L. X. Kang, J. T. Di, Y. Y. Zhang, and Q. W. Li, "Control synthesis and applications of carbon nanotube arrays," Chinese Science Bulletin, vol. 59, pp. 3264-3279, 2014.

[20] K. S. Novoselov, A. K. Geim, S. V. Morozov et al., "Electric field effect in atomically thin carbon films," Science, vol. 306, no. 5696, pp. 666-669, 2004.

[21] Y. C. Si and E. T. Samulski, "Synthesis of water soluble graphene," Nano Letters, vol. 8, no. 6, pp. 1679-1682, 2008.

[22] M. Sun and J. H. Li, "Graphene oxide membranes: Functional structures, preparation and environmental applications," Nano Today, vol. 20, pp. 121-137, 2018.

[23] J. Mohanraj, D. Durgalakshmi, R. A. Rakkesh, S. Balakumar, S. Rajendran, and H. Karimi-Maleh, "Facile synthesis of paper based graphene electrodes for point of care devices: a double stranded DNA (dsDNA) biosensor," Journal of Colloid and Interface Science, vol. 566, pp. 463-472, 2020.

[24] H. W. Kim, H. W. Yoon, S. M. Yoon et al., "Selective gas transport through few-layered graphene and graphene oxide membranes," Science, vol. 342, no. 6154, pp. 91-95, 2013.

[25] H. Li, Z. Song, X. Zhang et al., "Ultrathin, molecular-sieving graphene oxide membranes for selective hydrogen separation," Science, vol. 342, no. 6154, pp. 95-98, 2013.

[26] Y. H. Yang, L. Boiling, M. A. Priolo, and J. C. Grunlan, "Super gas barrier and selectivity of graphene oxide-polymer multilayer thin films," Advanced Materials, vol. 25, no. 4, pp. 503508, 2013.

[27] S. P. Koenig, L. Wang, and J. Pellegrino, "Selective molecular sieving through porous graphene," Nature Nanotechnology, vol. 7, no. 11, pp. 728-732, 2012.

[28] K. Celebi, J. Buchheim, R. M. Wyss et al., "Ultimate permeation across atomically thin porous graphene," Science, vol. 344, no. 6181, pp. 289-292, 2014.

[29] J. Shen, G. P. Liu, K. Huang, W. Jin, K. R. Lee, and N. Xu, "Membranes with fast and selective gas-transport channels of laminar graphene oxide for efficient CO2Capture," Angewandte Chemie, vol. 127, no. 2, pp. 588-592, 2015.

[30] A. S. Huang, Q. Liu, N. Y. Wang, Y. Q. Zhu, and J. Caro, "Bicontinuous zeolitic imidazolate framework ZIF-8@GO membrane with enhanced hydrogen selectivity," Journal of the American Chemical Society, vol. 136, no. 42, pp. 1468614689, 2014.

[31] C. Athanasekou, M. Pedrosa, T. Tsoufis, and L. M. PastranaMartínez, "Comparison of self-standing and supported graphene oxide membranes prepared by simple filtration: gas and vapor separation, pore structure and stability," Journal of Membrane Science, vol. 522, pp. 303-315, 2017.

[32] Y. Orooji, M. Faghih, A. Razmjou et al., "Nanostructured mesoporous carbon polyethersulfone composite ultrafiltration membrane with significantly low protein adsorption and bacterial adhesion," Carbon, vol. 111, pp. 689-704, 2017. 
[33] Y. Orooji, F. Liang, A. Razmjou et al., "Excellent biofouling alleviation of thermoexfoliated vermiculite blended Poly(ether sulfone) ultrafiltration membrane," ACS Applied Materials \& Interfaces, vol. 9, no. 35, pp. 30024-30034, 2017.

[34] L. L. Jiang, C. T. Li, H. T. Yu, Z. Zou, F. Shen, and X. Hou, "Preparation of $\mathrm{NH}_{2}-\mathrm{SH}-\mathrm{GO} / \mathrm{MWCNT}$ composite for simultaneous removal of $\mathrm{Pb}$ (II), $\mathrm{Zn}$ (II) and phenol from aqueous solution," Desalination and Water Treatment, vol. 97, pp. 272-284, 2017.

[35] M. L. Chua, Y. C. Xiao, and T. S. Chung, "Effects of thermally labile saccharide units on the gas separation performance of highly permeable polyimide membranes," Journal of Membrane Science, vol. 415-416, pp. 375-382, 2012.

[36] K. Chan, L. E. Kostun, and W. E. Tenhaeff, "Initiated chemical vapor deposition of polyvinylpyrrolidone-based thin films," Polymer, vol. 47, no. 20, pp. 6941-6947, 2006.

[37] B. H. Yang, J. R. Li, J. F. Wang, H. Xu, and C. Li, "Poly(vinyl pyrrolidone-co-octavinyl polyhedral oligomeric silsesquioxane) hybrid Nanocomposites: preparation, thermal properties, and T-g improvement mechanism," Journal of Applied Polymer Science, vol. 111, no. 6, pp. 2963-2969, 2009.

[38] Y. Zhao, B. T. Jung, and L. Ansaloni, "Multiwalled carbon nanotube mixed matrix membranes containing amines for high pressure $\mathrm{CO}_{2} / \mathrm{H}_{2}$ separation," Journal of Membrane Science, vol. 459, no. 2, pp. 233-243, 2014.

[39] A. I. Aria and M. Gharib, "Reversible tuning of the wettability of carbon nanotube arrays: the effect of ultraviolet/ozone and vacuum pyrolysis treatments," Langmuir, vol. 27, no. 14, pp. 9005-9011, 2011.

[40] L. Li, C. W. Song, D. W. Jiang, and T. Wang, "Preparation and enhanced gas separation performance of carbon/carbon nanotubes (C/CNTs) hybrid membranes," Separation \& Purification Technology, vol. 188, pp. 73-80, 2017.

[41] D. Y. Wu, C. H. Yi, and N. N. Lu, "Preparation and gas separation performance of PVAm/PVA/MWCNT mixed matrix membrane," Journal of Membrane Science, vol. 37, pp. 53-64, 2017.

[42] M. Amirilargani and B. Sadatnia, "Poly(vinylalcohol)/zeolitic imidazolate frameworks (ZIF-8) mixed matrix membranes for pervaporation dehydration of isopropanol," Journal of Membrane Science, vol. 469, pp. 1-10, 2014.

[43] D. M. Khan, A. Kausar, and S. M. Salman, "Buckypapers of polyvinyl chloride/poly (styrene-co-maleic anhydride) blend intercalated graphene oxide-carbon nanotube nanobifiller: physical property exploration," Fullerenes, Nanotubes and Carbon Nanostructures, vol. 24, no. 3, pp. 202-212, 2016.

[44] J. X. Wu, H. Xu, and J. Zhang, "Raman spectroscopy of graphene," Acta Chim Sinica, vol. 72, no. 3, pp. 301-318, 2014.

[45] J. Lee, H. R. Chae, Y. J. Won, and K. Lee, "Graphene oxide nanoplatelets composite membrane with hydrophilic and antifouling properties for wastewater treatment," Journal of Membrane Science, vol. 448, pp. 223-230, 2013.

[46] D. X. Yang, A. Velamakanni, G. Bozoklu et al., "Chemical analysis of graphene oxide films after heat and chemical treatments by X-ray photoelectron and micro-Raman spectroscopy," Carbon, vol. 47, no. 1, pp. 145-152, 2009.

[47] D. Graf, F. Molitor, and K. Ensslin, "Spatially resolved Raman spectroscopy of single-and-few-layer graphene," Nano Letters, vol. 7 , no. 2, pp. 238-242, 2007.
[48] S. Kim, L. Chen, J. K. Johnson, and E. Marand, "Polysulfone and functionalized carbon nanotube mixed matrix membranes for gas separation: theory and experiment," Journal of Membrane Science, vol. 294, no. 1-2, pp. 147-158, 2007.

[49] A. D. Kiadehi, M. Jahanshahi, and R. Ahmad, "The effect of functionalized carbon nano-fiber (CNF) on gas separation performance of polysulfone (PSf) membranes," Chemical Engineering \& Processing Process Intensification, vol. 90, pp. 41-48, 2015.

[50] A. D. Kiadehi, R. Ahmad, and M. Jahanshahi, "Novel carbon nano-fibers (CNF)/polysulfone (PSf) mixed matrix membranes for gas separation," Journal of Industrial and Engineering Chemistry, vol. 22, pp. 199-207, 2015.

[51] K. Zahri, K. C. Wong, P. Goh, and A. F. Ismail, “Graphene oxide/polysulfone hollow fiber mixed matrix membranes for gas separation," RSC Advances, vol. 6, no. 92, pp. 89130-89139, 2016.

[52] M. Sarfraz and M. Ba-Shammakh, "Synergistic effect of incorporating ZIF-302 and graphene oxide to polysulfone to develop highly selective mixed-matrix membranes for carbon dioxide separation from wet post-combustion flue gases," Journal of Industrial \& Engineering Chemistry, vol. 36, pp. 154-162, 2016.

[53] M. Sarfraz and M. Ba-Shammakh, "Synergistic effect of adding graphene oxide and ZIF-301 to polysulfone to develop high performance mixed matrix membranes for selective carbon dioxide separation from post combustion flue gas," Journal of Membrane Science, vol. 514, pp. 35-43, 2016. 